Este libro forma parte del acervo de la Biblioteca Jurídica Virtual del Instituto de Investigaciones Jurídicas de la UNAM

\title{
¿CÓMO SALVAR MÁS VIDAS GUANDO LOS REGURSOS SON ESCASOS? UNA ALTERNATIVA ANTE LA COVID-19
}

\author{
Adrián SOTO MOTA \\ Andrés Castañeda PRado \\ Luis F. FERNÁNDEZ RUIZ \\ Javier MARTÍN REYES
}

\begin{abstract}
SUMARIO: I. Introducción. II. La estrategia gubernamental de respuesta a la COVID-19. III. El problema y una posible solución. IV. Metodología para la estimación del modelo. V. ¿Es ético implementar criterios de triage?. VI. ¿Es constitucional implementar el modelo de triage propuesto?. VII. Conclusiones.
\end{abstract}

\section{INTRODUCGIÓN}

El origen de la pandemia por la COVID-19 en México inició un debate público sobre qué hacer ante la posible saturación del sistema de salud con recursos materiales, económicos y humanos limitados para la atención. El Consejo de Salubridad General, el máximo órgano en materia de salubridad en el país, ${ }^{1}$ encomendó en marzo a un Comité de Ética la integración de una propuesta de priorización para la asignación de recursos escasos.

El 11 de abril, horas antes de la aparición de la primera versión de la Guía Bioética para Asignación de Recursos Limitados de Medicina Crítica en Situación de Emergencia del Comité de Ética, ${ }^{2}$ se publicó una propuesta de asig-

\footnotetext{
1 Sobre los antecedentes y estatus constitucional del Consejo de Salubridad General, véase Cossío, José Ramón et al., "Reflexiones constitucionales sobre el Consejo de Salubridad General”, Gaceta Médica de México, México, núm. 149, 2013, pp. 356-362; así como Cossío Díaz, José Ramón y Sánchez Mejía, David, "El Consejo de Salubridad General en la Constitución mexicana de 1917”, en Gutiérrez Domínguez, Fernando (coord.), Secretaría de Salud: La salud en la Constitución mexicana, México, Secretaría de Cultura, INEHRM, Secretaría de Salud, 2017, pp. 73-92.

2 La primera versión del la Guía Bioética se publicó en la cuenta de Twitter del Consejo de Salubridad General (@DeSalubridad) a las 17:49 horas del 11 de abril de 2020, con el
} 
Este libro forma parte del acervo de la Biblioteca Jurídica Virtual del Instituto de Investigaciones Jurídicas de la UNAM www.juridicas.unam.mx

nación de recursos escasos en la revista Nexos, con el título "Un dilema inevitable del COVID-19: ¿Cómo salvar más vidas cuando se acaben las camas?". ${ }^{3}$ Este capítulo propone una versión más integral de la propuesta, así como una evaluación a la luz de la evidencia que se ha generado durante los últimos meses. ${ }^{4}$

La propuesta parte de dos hechos indiscutibles. Primero, México es uno de los países con mayor número de personas infectadas por el SARSCoV-2 (o COVID-19) y por lo tanto se necesita un número importante de camas de terapia intensiva. Segundo, pese a todo esfuerzo gubernamental, las camas de terapia intensiva y el personal médico para atenderlas han sido insuficientes. Frente a este escenario que ha cobrado la vida de más de 50 mil personas según las cifras oficiales,${ }^{5}$ hace evidente la necesidad de contar con parámetros objetivos, transparentes y equitativos para la asignación de recursos por parte de las autoridades sanitarias y el personal médico. Asimismo, en caso de existir criterios más efectivos, convendría actualizar los existentes.

La capacidad de atención del sistema de salud mexicano ha tenido retos y limitaciones importantes durante la pandemia. Esto ha sucedido a pesar de

siguiente mensaje: "El CSG comparte la Guía Bioética de Asignación de Recursos de Medicina Crítica, coordinada por los doctores María de Jesus Medina y Cesar Palacios González del Comité de Ética del Consejo, para hacer frente a la pandemia de COVID-19”. Hasta donde tenemos conocimiento, se publicaron tres versiones preliminares y una versión final de la guía, en las siguientes fechas y con diferentes denominaciones: Medina Arellano, María de Jesús et al., "Guía Bioética de Asignación de Recursos de Medicina Crítica", 11 de abril de 2020, disponible en: https://bit.ly/CSG_GuiaOriginal; Medina Arellano, María de Jesús et al., "Proyecto de Guía de Triaje para la Asignación de Recursos de Medicina Crítica", 15 de abril de 2020, disponible en: https://bit.ly/CSG_GuiaV2; Medina Arellano, María de Jesús et al., "Proyecto de Guía para Asignación de Recursos Médicos en Situación de Contingencia", 20 de abril de 2020, disponible en: https://bit.ly/CSG_GuiaV3; Consejo de Salubridad General, "Guía Bioética para Asignación de Recursos Limitados de Medicina Crítica en Situación de Emergencia”, 30 de abril de 2020, disponible en: https:// bit.ly/CSG_GuiaVFinal.

3 Soto Mota, Adrián et al., "Un dilema inevitable del COVID-19: ¿Cómo salvar más vidas cuando se acaben las camas?", Nexos, 11 de abril de 2020, disponible en: https://bit.ly/ Dilema_Inevitable. El presente capítulo está construido a partir de este artículo, así como de estos trabajos posteriores: Castañeda, Andrés et al., "México necesita una política clara de acceso a terapia intensiva", Etcétera, 16 de abril de 2020, disponible en: https://bit.ly/Politica_TI; así como Martín Reyes, Javier, "COVID-19, tratos diferenciados y test de escrutinio estricto", en González Martín, Nuria (coord.), Emergencia sanitaria por COVID-19. Reflexiones desde el derecho (III), México, UNAM, Instituto de Investigaciones Jurídicas, 2020, pp. 77-83, disponible en: https://bit.ly/Covid_Escrutinio.

4 Finalizamos la redacción de este texto el 7 de agosto de 2020.

5 Secretaría de Salud, "Informe Técnico Diario COVID-19 México", 6 de agosto de 2020, disponible en: https://bit.ly/3knXVwef. 
Este libro forma parte del acervo de la Biblioteca Jurídica Virtual del Instituto de Investigaciones Jurídicas de la UNAM

que el gobierno mexicano ha tomado medidas para aumentar la capacidad de atención: comprar más ventiladores mecánicos, ampliar la capacidad de camas disponibles, capacitar al personal médico que pueda atender a pacientes con SARS-GoV-2 y finalmente instalar sistemas de acompañamiento de especialistas para ampliar dicha capacidad.

A pesar de estos esfuerzos, el sistema de salud de algunas regiones y hospitales del país se ha visto rebasado. ${ }^{6}$ Aún con la reconversión de camas y su instalación sabemos que en algunas regiones y hospitales de salud las camas se terminaron. De acuerdo con datos preliminares de Sánchez-Talanquer, sólo el 1.2\% de pacientes con COVID-19 ingresaron a cuidados intensivos en el Instituto Mexicano del Seguro Social (IMSS), además de que ésta es la institución que reporta el mayor porcentaje de mortalidad, con el 43\% de las personas que fueron hospitalizadas por COVID-19 (véanse las figuras 1 y 2). ${ }^{7}$ Para poner en perspectiva estos datos y facilitar su comparación, tomemos en cuenta que se ha reportado que entre el $5 \%$ y el $16 \%$ de los pacientes hospitalizados por COVID requieren atención en terapia intensiva. Esto invita a pensar que al no contar con camas suficientes no se les pudo brindar toda la atención necesaria a los derechohabientes y que, en general, las autoridades han buscado limitar el acceso a las camas, en tanto la ocupación hospitalaria es la variable más relevante para los semáforos epidemiológicos que se han implementado.

Ante una pandemia como la que enfrentamos, el personal médico tiene que tomar importantes decisiones: ¿A quién otorga los recursos escasos (cualquier combinación entre camas, ventiladores y personal capacitado)? ¿En qué momento retirar los respiradores a pacientes que no mejoran a pesar de la atención o para priorizar a pacientes con mayor probabilidad de vivir? ¿Cómo tomar esas decisiones bajo un contexto de presión, riesgo legal y donde todas las personas exigen la atención para sí mismos o para sus familiares? ¿Cómo salvar el mayor número de vidas, bajo el supuesto de que todas son igualmente dignas y valiosas?

6 Romo, Gerardo, "Se agotan camas con ventilador en IMSS para pacientes COVID-19", Ágora Digital, 6 de agosto de 2020, disponible en: https://bit.ly/2Py4xtA; Uscanga, Oscar. "Agota Yucatán camas COVID; habilita hospitales temporales", Mural, 6 de agosto de 2020, disponible en: https://bit.ly/31v5mt1.

7 Sánchez-Talanquer, Mariano, "Desigualdad en la pandemia: Evidencia de México", documento de trabajo, 2020 (las gráficas se encuentran disponibles en: https://bit.ly/ MST_Pandemia). 
Este libro forma parte del acervo de la Biblioteca Jurídica Virtual del Instituto de Investigaciones Jurídicas de la UNAM

\section{FIGURA 1}

Porcentaje de pacientes con COVID-19

que ingresaron a cuidados intensivos, por institución

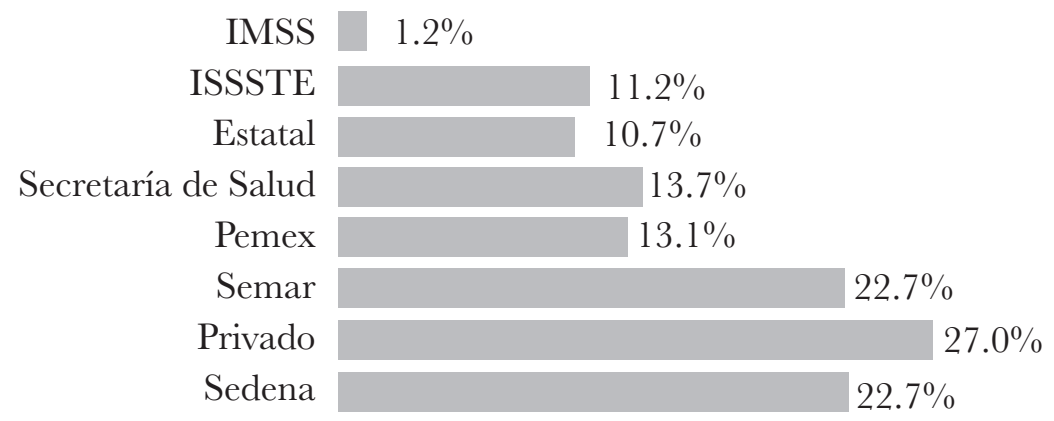

FuENTE: Sánchez-Talanquer (2020). ${ }^{8}$

FIGURA 2

Mortalidad COVID-19, por institución

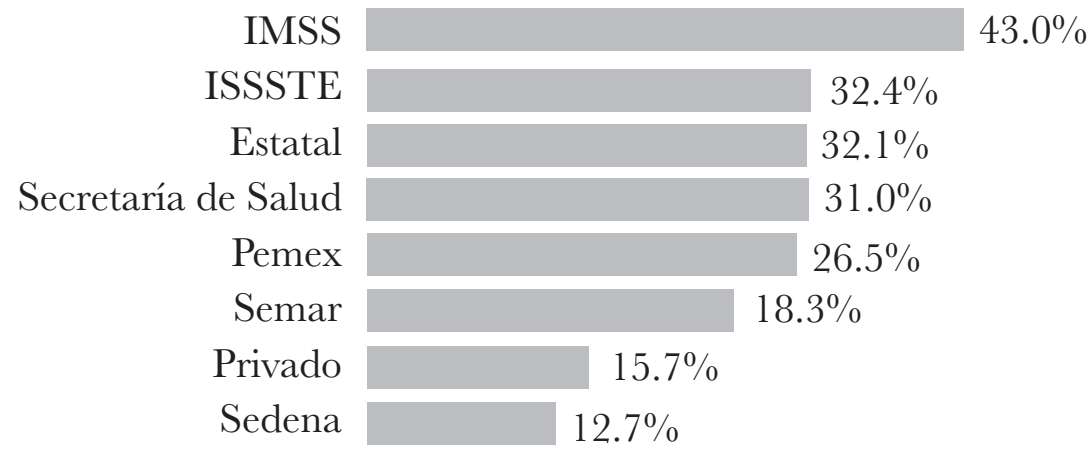

FuENTE: Sánchez-Talanquer (2020). ${ }^{9}$

8 Idem. Para información adicional de contexto consultar: Grasselli, Giacomo et al., "Critical Care Utilization for the COVID-19 Outbreak in Lombardy, Italy", Fournal of the American Medical Association, vol. 323, núm. 15, 2020, pp. 1545 y 1546, disponible en: https:// bit.ly/31tveW9.

9 Idem. 
Este libro forma parte del acervo de la Biblioteca Jurídica Virtual del Instituto de Investigaciones Jurídicas de la UNAM

La decisión no es fácil y la respuesta tiene límites técnicos, éticos y jurídicos que aquí discutimos. La solución a muchas de estas interrogantes pasa por la implementación de estrategias de triaje (triage),${ }^{10}$ un método que utiliza criterios clínicos para definir el ingreso de pacientes con COVID-19 a terapia intensiva, lo cual reduce la discrecionalidad en la toma de decisiones médicas y evita los criterios aleatorios de asignación como el de "el primero en llegar, primero en ser atendido". Este tipo de estrategia no es una solución nueva. Se utiliza en las salas de urgencias y en las zonas de desastre en donde muchas veces se enfrentan dilemas similares. Un buen ejemplo es la lista de donadore ${ }^{11}$ en donde se tienen recursos limitados y se prioriza a los pacientes que mayor beneficio recibirán del órgano.

Ante estos dilemas, desde abril de 2020 presentamos una propuesta con la intención de garantizar la priorización y de salvar el mayor número de vidas posibles. En particular, propusimos un modelo específico que permitiría a los profesionales de salud adoptar criterios de triage para identificar a aquellos pacientes que más se benefician con cuidados especializados y así priorizar su atención. Con este modelo es posible salvar la mayor cantidad de vidas posible (asumiendo que todas las vidas valen lo mismo); evitar la discriminación por condiciones pre-existentes a la situación de salud actual; reducir la carga de trabajo y la carga emocional que este proceso impone en el personal de salud a cargo de tomar estas decisiones; y blindar jurídicamente las decisiones del personal de salud.

Para hacer una asignación de este tipo es necesario tener un modelo que permita predecir qué pacientes tienen una mayor probabilidad de sobrevivir en terapia intensiva. En este texto presentamos el modelo que propusimos al Estado mexicano y que tiene la ventaja de emplear variables independientes y usualmente disponibles en todos los hospitales mexicanos que atienden pacientes con COVID-19. Como se mostrará, este modelo se

10 Triage es un término de origen francés (del verbo trier, cribar u ordenar) y un término militar que significa seleccionar, escoger o priorizar. En la actualidad, se utiliza en medicina, especialmente en la de emergencia y de desastres, para hacer referencia a los procedimientos de selección y priorización, entre otras cosas, de las posibilidades de supervivencia.

11 Esta lógica tiene, incluso, cobertura legal en el ordenamiento jurídico mexicano. El artículo 336, párrafo primero, de la Ley General de Salud señala que "[p]ara la asignación de órganos y tejidos de donador no vivo, se tomará en cuenta la gravedad del receptor, la oportunidad del trasplante, los beneficios esperados, la compatibilidad con el receptor y los demás criterios médicos aceptados, así como la ubicación hospitalaria e institucional del donador". Por su parte, el segundo párrafo del mismo artículo enfatiza que solo "[c]uando no exista urgencia o razón médica para asignar preferentemente un órgano o tejido, ésta se sujetará estrictamente a las bases de datos hospitalarias, institucionales, estatales y nacional que se integrarán con los datos de los pacientes registrados en el Centro Nacional de Trasplantes". 
Este libro forma parte del acervo de la Biblioteca Jurídica Virtual del Instituto de Investigaciones Jurídicas de la UNAM

justifica en términos médicos, éticos y jurídicos y sirve para salvar vidas en esta ola de la pandemia y en las subsecuentes. Además ayuda a prevenir la discriminación y la desigualdad en el acceso a la atención médica.

A continuación, presentamos un panorama general sobre la estrategia gubernamental sugerida para incrementar el número de camas. Posteriormente presentamos la metodología a partir de la cual se estimó el modelo. Finalmente, reflexionamos sobre las justificaciones éticas y jurídicas de la propuesta.

\section{LA ESTRATEGIA GUBERNAMENTAL DE RESPUESTA A LA COVID-19}

El gobierno ha seguido una estrategia con tres fases dirigidas a expandir la capacidad de atención a pacientes infectados por SARS-CoV-2. ${ }^{12}$ En la FASE-1, se buscó aumentar la disponibilidad de camas de terapia intensiva que ya existían (cancelando cirugías electivas, por ejemplo). Es importante notar que las camas previamente existentes (alrededor de 1,200), ${ }^{13}$ además de tener el equipo y la infraestructura adecuados para la atención de pacientes críticos, ya contaban con personal de salud experimentado en el manejo de pacientes con Síndrome de Insuficiencia Respiratoria Aguda (SIRA), la forma más grave de infección por el virus SARS-CoV-2. ${ }^{14}$

En la FASE-2, camas y personal de salud que habitualmente no participan en la atención de pacientes críticos se "convirtieron" o adaptaron en camas de terapia intensiva, añadiendo alrededor de 5,200 camas adicionales. ${ }^{15}$ Sin embargo, el principal déficit de nuestro sistema de salud es el de recursos humanos. Para afrontarlo, se diseñó un sistema "atención en cascada" 16 en el que especialistas en medicina crítica apoyarán resolviendo

12 Secretaría de Salud, Lineamiento de Reconversión Hospitalaria, versión 5 de abril, 2020, disponible en: https://bit.ly/2PAFdn6.

13 Roldán, Nayeli, "Habrá 'reconversión hospitalaria' para atender a pacientes graves por COVID-19 con 1,283 camas", Animal Politico, 30 de marzo de 2020, disponible en: https://bit.ly/3kohCEe.

14 Zhou, Fei et al., "Clinical course and risk factors for mortality of adult inpatients with COVID-19 in Wuhan, China: a retrospective cohort study", Lancet, vol. 396, 28 de marzo de 2020, disponible en: https://bit.ly/31vUriv.

15 Baranda, Antonio y Guerrero, Claudia, "Tiene México 6,425 camas para COVID-19", Reforma, 5 de abril de 2020, disponible en: https://bit.ly/3ïDBKz.

16 Presidencia de la República, "Versión estenográfica. Conferencia de prensa. Informe diario sobre coronavirus COVID-19 en México", 4 de abril de 2020, disponible en: https:// bit.ly/3illXad. 
Este libro forma parte del acervo de la Biblioteca Jurídica Virtual del Instituto de Investigaciones Jurídicas de la UNAM

las dudas de cinco médicos generales o de otras especialidades y estarán a cargo de veinticinco pacientes en total.

La tercera fase contempló la adaptación de espacios enteros (hospitales inflables, gimnasios y hoteles) para la atención de enfermos con COVID-19. ${ }^{17}$

\section{EL PROBLEMA Y UNA POSIBLE SOLUCIÓN}

Comparado con otros países, México enfrenta la pandemia con un número muy reducido de camas de terapia intensiva por cada 100,000 habitantes. Incluso si la estrategia de reconversión hospitalaria fuera exitosa en instalar 5,200 camas adicionales para conseguir un total de 6,400 camas, México contaría con 0.06 camas por cada 100,000 habitantes. Si comparamos esta cifra con las 34.7 de Estados Unidos, las 29.2 de Alemania, las 12.5 de Italia o las 3.6 de China y las 2.3 de la India, es claro que México tiene un déficit notable (véase la Figura 3).

\section{FIGURA 3. LOS PAÍSES CON MÁS GAMAS DE CUIDADOS INTENSIVOS PER CÁPITA}

Número total de camas de cuidados intensivos por cada 100 mil habitantes en países seleccionados

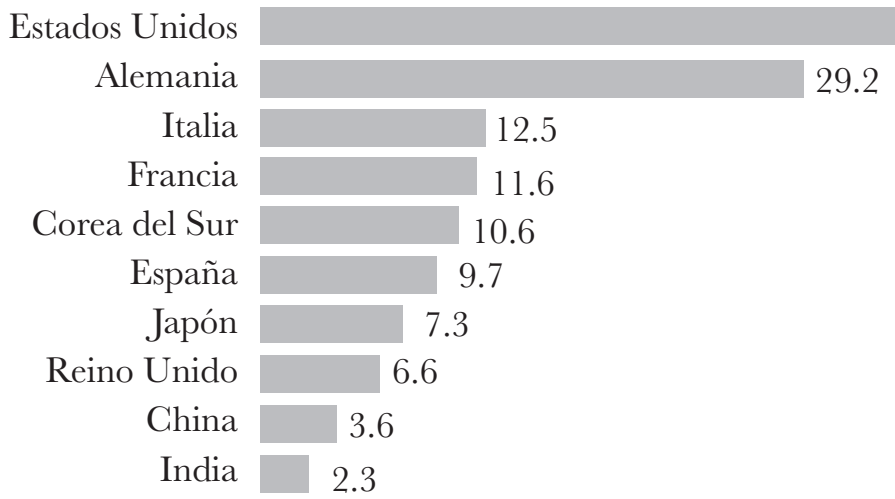

FuENTE: McCarthy (2020). ${ }^{18}$

\footnotetext{
17 Secretaría de Salud, op. cit.

18 McCarthy, Niall, "The Countries With The Most Critical Care Beds Per Capita", Forbes, 12 de marzo de 2020, disponible en: https://bit.ly/2DMinpC.
} 
Este libro forma parte del acervo de la Biblioteca Jurídica Virtual del Instituto de Investigaciones Jurídicas de la UNAM

Tomando en cuenta que sistemas de salud con mucho mejor infraestructura para la atención de pacientes críticos se vieron rebasados durante la pandemia, no es sorprendente que las camas de FASE-1 destinadas a la atención de pacientes críticos hayan resultado insuficientes.

Además, vale la pena subrayar que (en igualdad de condiciones clínicas) el pronóstico de un paciente crítico hospitalizado en una cama de FASE-1 es claramente mejor que el de un paciente hospitalizado en una cama de FASE-2 (camas convertidas o adaptadas), ya que en las primeras se cuenta con personal más experimentado y una infraestructura ya establecida.

Además, a pesar de que las cifras oficiales reportan disponibilidad de camas de terapia intensiva en todos los estados, la escasez de camas ha sido una realidad. Muestra de ello es la diferencia en mortalidad y acceso a terapia intensiva que se ha observado en los diferentes sistemas de salud durante la pandemia (véanse las figuras 1 y 2). Debido a que la demanda que tiene cada sistema de salud es diferente, hay escasez de camas en algunos hospitales aunque no la haya en otros. Por lo tanto, un sistema para guiar la distribución de recursos materiales y humanos, sigue siendo necesario.

La escasez de recursos materiales y humanos para la atención de pacientes críticos con COVID-19 ya ha ocurrido en otros países. Pasó en Italia, Francia, España, Reino Unido y ahora pasa en los Estados Unidos en donde hospitales en diversos estados como New York, Alabama, Arizona, Kansas, Louisiana, Maryland, Michigan, New York, Pennsylvania, Tennessee, Utah y Washington State han implementado sus propios sistemas de triage. ${ }^{19}$ Diversas instituciones de salud en todo el mundo han diseñado distintos criterios para optimizar equitativamente ${ }^{20}$ la asignación ${ }^{21}$ de pacientes críticos en un contexto de recursos limitados y mejorar su probabilidad de tener un desenlace favorable. Sin embargo, dichos criterios emplean algunas herramientas (como algunos estudios de laboratorio) que no se utilizan rutinariamente en los hospitales mexicanos.

Es una obviedad, pero vale la pena enfatizarlo: no todos los sistemas de triage funcionan bajo el mismo criterio, incluso tratándose de la misma pato-

19 Emanuel, Ezekiel J. et al., "Fair Allocation of Scarce Medical Resources in the Time of COVID-19", The New England Fournal of Medicine, vol. 382, 21 de mayo de 2020, pp. 20492055, disponible en: https://bit.ly/31v2s7r.

20 Grupo de Trabajo de Bioética de la SEMICYUC, "Recomendaciones éticas para la toma de decisiones en situación excepcional de crisis por pandemia COVID-19 en las unidades de cuidados intensivos (SEMICYUC)", Sociedad Española de Medicina Intensiva, Crítica y Unidades Coronarias, 2020, disponible en: https://bit.ly/33KbcfP.

21 White, Douglas B. y Lo, Bernard, "A Framework for Rationing Ventilators and Critical Care Beds During the COVID-19 Pandemic", Fournal of the American Medical Association, vol. 323, núm. 18, 27 de marzo de 2020, pp. 1773 y 1774, disponible en: https://bit.ly/33COvqF. 
Este libro forma parte del acervo de la Biblioteca Jurídica Virtual del Instituto de Investigaciones Jurídicas de la UNAM

logía. El modelo a usar depende del contexto, los instrumentos de medición disponibles y, en gran medida, de los criterios éticos concretos. Por ejemplo, en Italia se decidió poner una edad límite para acceder a un ventilador mecánico ${ }^{22}$ y en el estado de Alabama (Estados Unidos) se sugiere que las personas con retraso mental severo no deberían ser los principales candidatos para ingresar a una terapia intensiva. ${ }^{23}$

También reconocemos que existen otros puntos de vista, según los cuales salvar al mayor número de vidas posibles no es el objetivo más adecuado. Se ha propuesto, por ejemplo, salvar la mayor utilidad en años de vida ajustados por calidad, es decir número de años libres de enfermedad. Incluso hay quienes consideran que los profesionales de salud deberían tener prioridad, ya que el salvarlos permitirá salvar a aún más personas. No obstante, consideramos que, bajo el principio de que toda vida es igualmente digna y valiosa, las autoridades deben salvar al mayor número de personas, empleando para ello un mecanismo de triage que considere más de un parámetro, que no sea discriminatorio y pueda ser implementado en todo el país de manera rápida y efectiva.

Con esto en mente, proponemos el uso de un modelo para estimar la mortalidad intrahospitalaria de las personas diagnosticadas con COVID-19 y su uso como auxiliar en la toma de decisiones de ingreso a terapia intensiva. A partir de este modelo, consideramos que las camas FASE-1 deberían ser asignadas a los pacientes que tengan una mayor probabilidad de supervivencia.

\section{METODOLOGÍA PARA LA ESTIMAGIÓN DEL MODELO}

Para calcular la probabilidad de fallecer durante la hospitalización de una persona con COVID, se desarrolló un modelo basado en el nomograma de Fagan para la concatenación bayesiana de varios factores pronósticos ya identificados. ${ }^{24}$ A continuación detallamos los pasos para ello:

22 Rosemaum, Lisa, "Facing COVID-19 in Italy - Ethics, Logistics, and Therapeutics on the Epidemic's Front Line", New England Fournal of Medicine, vol. 382, 2020, pp. 18731875, disponible en: https://bit.ly/2DzWDxF.

23 State of Alabama, "Criteria for Mechanical Ventilator Triage Following Proclamation of Mass-Casualty Respiratory Emergency", 7 de agosto de 2020, disponible en: https://bit. ly $/ 2 P y T U q B$.

24 Fagan, T. J., "Nomogram for Bayes Theorem", The New England Fournal of Medicine, vol. 293, núm. 5, 1975, p. 257, disponible en: https://bit.ly/3gEbQMh. 
Este libro forma parte del acervo de la Biblioteca Jurídica Virtual del Instituto de Investigaciones Jurídicas de la UNAM

1. Se recolectó la razón de probabilidad (LR) publicados hasta la fecha de los factores que predicen la mortalidad de pacientes hospitalizados por SARS-CoV-2 ${ }^{25}$ y se agruparon por independencia fisiopatológica (es decir que no estuvieran relacionados entre ellos como por ejemplo fumar e infartarse).

2. Se modelaron varias combinaciones de variables y se escogió la combinación de variables que permitiera utilizar la mayor cantidad de variables independientes y que, con mayor probabilidad, estarán disponibles en todos los hospitales que atiendan pacientes con COVID-19. Estas fueron: i) edad, ii) saturación de oxígeno en la sangre (SpO2), iii) diagnóstico previo de hipertensión arterial sistémica, iv) elevación de enzimas cardiacas, v) conteo total de leucocitos, vi) conteo de linfocitos y vii) elevación de la creatinina. ${ }^{26} \mathrm{~A}$ la combinación de estas variables se les denominó LOW-HARM, por sus siglas en inglés.

3. Utilizando como probabilidad pre-prueba la probabilidad de fallecimiento observada por grupo etario en el mundo, ${ }^{27} \mathrm{se}$ automatizó el cálculo de la siguiente manera:

1. Odds-preprueba $=$ Probabilidad preprueba $/(1$ - Probabilidad preprueba).

2. Odds-postprueba $=($ Odds preprueba $) \times($ LR SpO2 $) \times($ Diagnóstico previo de Hipertensión Arterial Sistémica) x (LR elevación de enzimas cardiacas) $\mathrm{x}$ (LR conteo de leucocitos totales $>10000) \mathrm{x}$ (LR conteo de linfocitos > 0.8) x (LR elevación de la creatinina). Si el factor de riesgo está ausente, se multiplica por 1 y, de este modo, la probabilidad no cambia.

3. Probabilidad postprueba $=$ Odds postprueba $/(1+$ Odds postprueba).

La figura 4, ejemplifica el cálculo de un caso hipotético:

25 Zhou, op. cit.; Ruan, Qiurong et al., "Clinical predictors of mortality due to COVID-19 based on an analysis of data of 150 patients from Wuhan, China", Intensive Care Med, vol. 46, núm. 5, 3 de marzo de 2020, disponible en: https://bit.ly/31v7kcO.

26 Likelyhood-ratio: $\mathrm{SpO}_{2}>90 \%=0.897$, Diagnóstico previo de HAS=3.05, elevación de enzimas cardiacas $=10$, leucocitos totales $>10000 \times 10^{9} / L^{*}=6.6$, linfocitos $>0.8 \times 10^{9} /$ $\mathrm{L}=0.02$, elevación de creatinina sérica $=4.39$ (Zhou, op. cit.).

27 Worldometers, "Age, Sex, Existing Conditions of COVID-19 Cases and Deaths", 12 de marzo de 2020, disponible en: https://wrww.worldometers.info/coronavirus/coronavirus-age-sexdemographics/. 
Este libro forma parte del acervo de la Biblioteca Jurídica Virtual del Instituto de Investigaciones Jurídicas de la UNAM

\section{FigURA 4. ILUSTRACIÓN DE UN GASO HIPOTÉTICO}

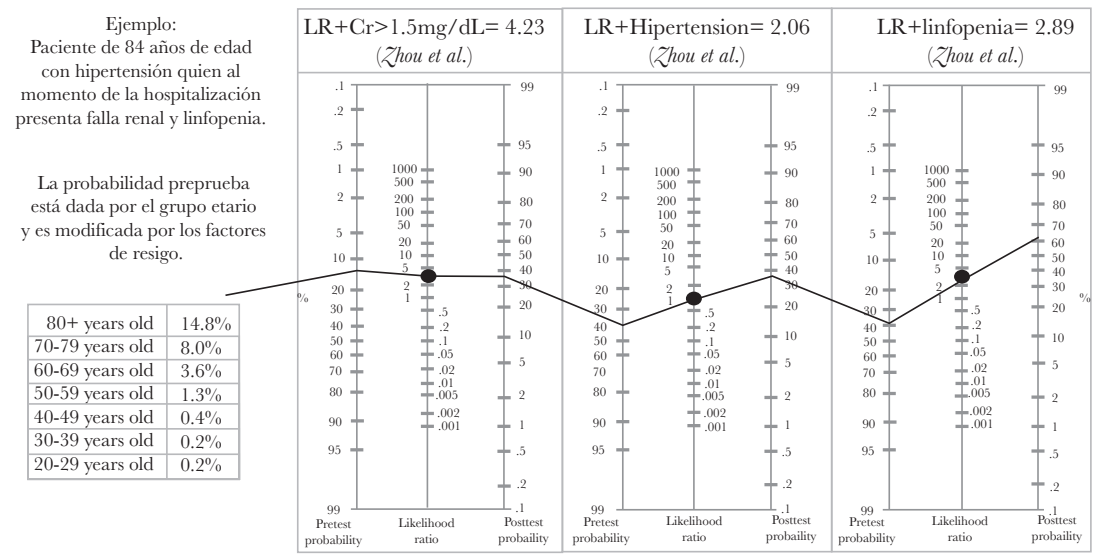

FUENTE: elaboración propia.

A continuación, se muestra la distribución de todas las combinaciones de factores de riesgo posibles para cada grupo etario ajustada para la prevalencia de hipertensión arterial sistémica para cada grupo etario en Méxi$\mathrm{co}^{28}$ La siguiente gráfica muestra que la simulación de resultados posibles permite tener certeza de que la edad es un factor contribuyente pero no determinante en el resultado final (ver la figura 5).

\section{Figura 5. DisTRibución DE TODOS LOS RESULTADOS POSIBLES}

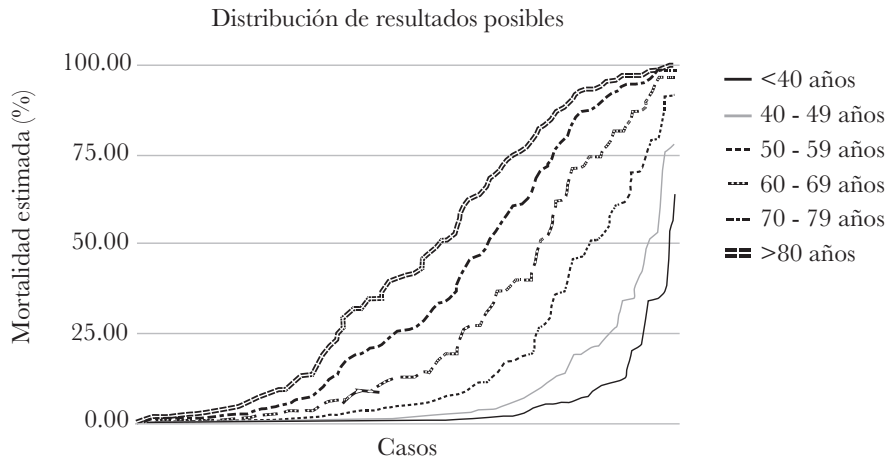

FUENTE: elaboración propia.

28 Rosas-Peralta, Martín et al., "Consenso de Hipertensión Arterial Sistémica en México", Revista Médica del Instituto Mexicano del Seguro Social, vol. 54, sup. 1, 23 de noviembre de 2015, disponible en: https://bit.ly/3iqyYyB. 
Este libro forma parte del acervo de la Biblioteca Jurídica Virtual del Instituto de Investigaciones Jurídicas de la UNAM

Con el fin de evaluar la capacidad pronóstica de este modelo y validarlo, se recolectó la información clínica al inicio de la hospitalización de 400 pacientes (200 egresos y 200 defunciones) hospitalizados en 12 instituciones en 10 diferentes ciudades del país. Se corroboró que el modelo propuesto tiene una excelente capacidad para diferenciar a los pacientes que fallecieron de aquellos que egresaron a su domicilio.

Además, se comparó el poder predictivo de este modelo con otros modelos propuestos para el mismo fin (figura 6) y se desarrolló una plataforma digital para facilitar los cálculos. Este análisis fue pre-registrado y aceptado para publicación en la revista del Colegio Americano de Medicina de Urgencias y en el Repositorio MedRxiv de Yale.

\section{FIGURA 6. COMPARACIÓN DEL DESEMPEÑO DE DISTINTOS MODELOS PREDICTIVOS PARA MORTALIDAD EN PACIENTES MEXICANOS CON COVID-19}

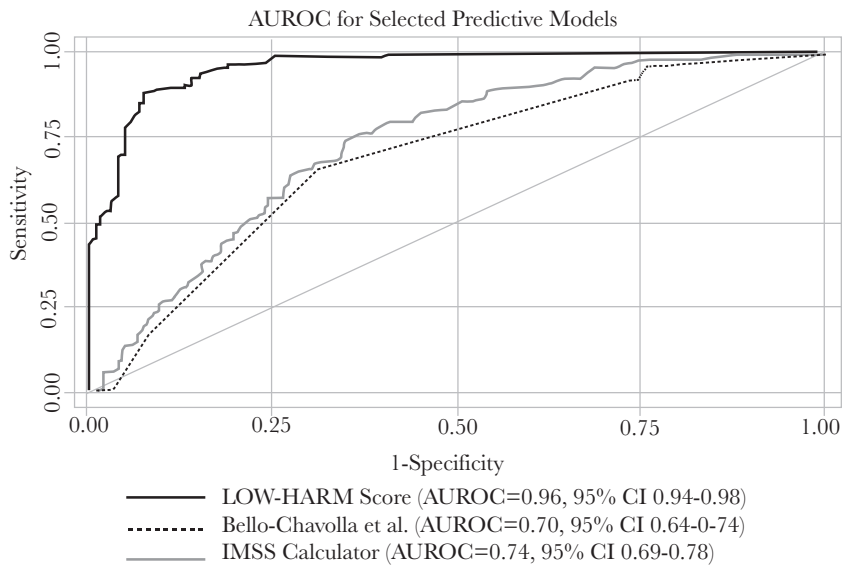

FuENTE: Soto-Mota et al. (2020). ${ }^{29}$

Más allá de los méritos técnicos de la propuesta, lo cierto es que el modelo de asignación que se propone también debe tomar en cuenta los deseos y expectativas del paciente y cuidar que implementación de una propuesta como ésta sea eficiente, práctica y generalizada. Asimismo, para su viabilidad es indispensable que los médicos a cargo de las unidades de terapia intensiva estén familiarizados con el sistema.

29 Soto-Mota, Adrián et al., "The Low-Harm Score for Predicting Mortality in Patients Diagnosed with COVID-19: A Multicentric Validation Study”, medRxiv, 5 de junio de 2020, disponible en: https://bit.ly/33FNxdw. 
Este libro forma parte del acervo de la Biblioteca Jurídica Virtual del Instituto de Investigaciones Jurídicas de la UNAM

Finalmente, en términos de implementación, conviene tener presente que hoy existe tecnología que permitiría tener en tiempo real los datos de la condición de cada paciente en el país de manera georeferenciada. Los médicos tratantes podrían actualizar los datos conforme cambie la condición de cada paciente y un centro de control podría asesorar en la gestión de camas. Las herramientas ya existen, los obstáculos para su implementación no son técnicos ni científicos.

\section{V. ¿ES ÉTICO IMPLEMENTAR GRITERIOS DE TRIAGE? ${ }^{30}$}

Independientemente de los aspectos técnicos de la propuesta, conviene preguntarse si criterios de triage como los propuestos son acordes con estándares éticos. Lo primero que habría que decir es que este tipo de situaciones excepcionales no son ajenas al campo de la salud. Se utilizan en situaciones de "medicina de desastres", aplicando una atención de crisis excepcional basada en la justicia distributiva y en la asignación adecuada de los recursos de salud. Además, este tipo de estrategias para la toma de decisiones tiene efectos positivos como reducir el desperdicio de recursos, evitar la pérdida de vidas, respaldar a los profesionales de salud y, sobre todo, evitar decisiones sesgadas y arbitrarias.

El síndrome de insuficiencia respiratoria aguda es una situación clínica de mucha gravedad. Hoy sabemos que, incluso en un entorno de abundancia, la mortalidad de los pacientes infectados por SARS-CoV-2 que requieren ventilación mecánica llega a ser hasta del $70 \%$ y el $86 \%,{ }^{31}$ lo que implica que sólo algunos pacientes se van a beneficiar del uso del soporte ventilatorio. Es a estos pacientes a quienes se debe priorizar en el acceso a los procedimientos necesarios.

Las decisiones difíciles son constantes para quienes están a cargo de los pacientes críticos. Facilitar criterios colegiados permite compartir la responsabilidad en estos escenarios que imponen una gran carga emocional sobre el personal de salud y da la confianza necesaria para que se puedan tomar las mejores decisiones.

Basar estas decisiones en la mejor evidencia científica disponible y promover su aplicación generalizada evita el uso de criterios únicos, arbitrarios

\footnotetext{
30 Agradecemos la discusión sobre esta sección a Luz Fojo Nava, Rodrigo Sánchez Castellanos y Alberto Toledo Urbina.

31 Namendys-Silva, Silvio A., "Respiratory support for patients with COVID-19 infection”, Lancet Respiratory Medicine, vol. 8, núm. 4, p. 8, 5 de marzo de 2020, disponible en: https://bit.ly/31zBPy8.
} 
Este libro forma parte del acervo de la Biblioteca Jurídica Virtual del Instituto de Investigaciones Jurídicas de la UNAM

y potencialmente estigmatizantes que no reflejan el verdadero potencial de recuperación y que ponen en desigualdad de condiciones a grupos vulnerables (como a los adultos mayores, a las personas que viven con VIH y a las personas en situación de calle).

El triage propuesto, además, se aplicaría de manera extraordinaria ante la situación actual, en sustitución de los modelos de triage vigentes durante lo que dure la pandemia o en los brotes posibles siguientes. Hay que enfatizar que estos esquemas no suponen el abandono de los pacientes, sino modificar los objetivos de su tratamiento al priorizar su acceso a los servicios de salud o al control de sus síntomas de acuerdo con sus probabilidades de sobrevivir.

En conclusión: adoptar, promover y transparentar criterios de triage que busquen aprovechar al máximo los recursos disponibles y que estén basados en evidencia evita que este tipo de decisiones difíciles sean resueltas por sesgos individuales en contextos de desigualdad estructural, conflictos de interés o actos de corrupción. Resuelve problemas de objetividad médica, reduce la posibilidad de inconformidad de pacientes y familiares (incluso por vías legales) e informa con claridad sobre la medida con la clara finalidad de salvar el mayor número de vidas posibles.

\section{VI. ¿ES CONSTITUGIONAL IMPLEMENTAR EL MODELO DE TRIAGE PROPUESTO? ${ }^{32}$}

La implementación de criterios de triage debe ser acorde con los estándares de derechos humanos. Sólo de esta forma se puede garantizar la constitucionalidad de ésta y otras medidas que tengan como finalidad el salvar el mayor número de vidas posibles. Incorporar una lógica de derechos humanos permite que el marco jurídico respalde adecuadamente las decisiones dificiles y los dilemas que enfrentan los profesionales de la salud y las autoridades del sistema de salud.

Un primer parámetro que debe cumplirse consiste en demostrar que el Estado mexicano ha utilizado todos sus recursos disponibles para ampliar su capacidad al máximo posible para garantizar los derechos a la salud y la vida de las personas en el contexto de la pandemia COVID-19. El triage podrá ser un instrumento constitucionalmente legítimo para salvar el mayor número de vidas posibles sólo si, de manera previa, el Estado mexicano

32 Agradecemos los comentarios que en su momento nos hicieron José Antonio Caballero Juárez, Juan Jesús Garza Onofre y Ximena Medellín Urquiaga. 
Este libro forma parte del acervo de la Biblioteca Jurídica Virtual del Instituto de Investigaciones Jurídicas de la UNAM

demuestra que le es imposible, en términos materiales, ampliar aún más su capacidad instalada (camas, ventiladores y personal capacitado).

Un segundo parámetro que debe cumplir la medida consiste en estar basada en criterios objetivos, transparentes y equitativos. En ese sentido, los criterios de triage propuestos realizan una asignación de camas de terapia intensiva escasas con criterios predefinidos que evalúan las probabilidades de supervivencia con base en siete criterios objetivos y transparentes: i) edad, ii) saturación de oxígeno en la sangre $\left(\mathrm{SpO}_{2}\right)$, iii) diagnóstico previo de hipertensión arterial sistémica, iv) elevación de enzimas cardiacas, $v$ ) conteo total de leucocitos, vi) conteo de linfocitos y vii) elevación de la creatinina sérica. Ello permite, de manera objetiva, definir probabilidades de supervivencia ante la COVID-19 para maximizar los beneficios posibles y salvar el mayor número posible de vidas. Asimismo, los criterios propuestos reducen la discrecionalidad en la toma de decisiones que hoy tienen los médicos o para evitar criterios inadecuados como el de "primero en llegar, primero en ser atendido".

La medida propuesta garantiza, además, el trato igualitario a las personas en todo el país. Ante la diversidad de subsistemas de salud y la heterogeneidad de unidades de cuidado intensivo en el país, y dada la adecuación de camas con ventiladores mecánicos y la capacitación en cascada de intensivistas para ampliar la capacidad de atención, se debe contar con criterios de priorización que razonablemente puedan ser implementados en todo el país. La homologación de los criterios de priorización fortalece la labor de los profesionales de la salud en la toma de decisiones, especialmente si son producto de decisiones colegiadas, pues permiten compartir la responsabilidad en decisiones complejas que deben de comunicar de manera efectiva a pacientes y familiares.

El tercer parámetro que debe cumplir la medida es superar un test de escrutinio estricto, lo que permite evitar que se torne discriminatoria. La aplicación de este nivel de escrutinio es necesario, pues el criterio de priorización es una medida que hace clasificaciones a partir de "categorías sospechosas" como la edad o las condiciones de salud. ${ }^{33}$

De acuerdo con la jurisprudencia obligatoria de la Suprema Corte de Justicia de la Nación (SCJN), esto implica que cualquier medida que utilice

33 Ambas categorías se encuentran contempladas en el párrafo quinto del artículo 10. constitucional: "Queda prohibida toda discriminación motivada por origen étnico o nacional, el género, la edad, las discapacidades, la condición social, las condiciones de salud, la religión, las opiniones, las preferencias sexuales, el estado civil o cualquier otra que atente contra la dignidad humana y tenga por objeto anular o menoscabar los derechos y libertades de las personas". 
Este libro forma parte del acervo de la Biblioteca Jurídica Virtual del Instituto de Investigaciones Jurídicas de la UNAM

este tipo de clasificaciones debe: i) cumplir una finalidad imperiosa desde el punto de vista constitucional, ii) estar estrechamente vinculada con la finalidad y iii) ser la medida menos restrictiva posible para conseguir efectivamente la finalidad. ${ }^{34}$

El modelo propuesto cumple con los tres requisitos. En primer lugar, la medida persigue finalidades imperiosas. El sistema de asignación busca incrementar el número de vidas salvadas en el contexto de la epidemia, esto es, busca garantizar de la manera más amplia posible los derechos constitucionales a la salud y a la vida. La SCJN ha reconocido en repetidas ocasiones que proteger la salud y vida de las personas es una finalidad constitucionalmente válida, que justifica incluso la afectación a derechos fundamentales o tratos diferenciados. ${ }^{35}$ Por supuesto, la consecución de estas finalidades se vuelve aún más urgente y apremiante en el contexto de la pandemia por la COVID-19.

En segundo lugar, existe una vinculación estrecha entre el triage propuesto y la finalidad de salvar el mayor número de vidas dados los recursos disponibles. Como se ha mostrado previamente, la combinación de criterios de priorización con la asignación efectiva de camas, ventiladores y personal capacitado maximiza la probabilidad de vida del mayor número de personas posibles y reduce al máximo problemas de sobre y sub inclusión en la asignación de camas de FASE-1 a la luz de esta finalidad.

En tercer lugar, la medida cumple con el parámetro de necesidad. Ante el escenario de saturación del sistema de salud, no existe otra medida igualmente efectiva para salvar el mayor número de vidas y que sea menos gravosa en terminos de igualdad (no discriminación) o de derechos. La existencia de otros modelos de priorización como el Apache o el SOFA para terapias intensivas no consideran las variables ni las condiciones específicas de la COVID-19, por lo que se requiere diseñar un nuevo triage que considere condiciones médicas específicas de riesgo y de necesidades de atención, así como la identificación de casos en todo el territorio mexicano. Asimismo, los criterios del triage propuestos evitan al máximo uso de clasificaciones basadas en enfermedades y precondiciones (personas que viven con VIH,

34 Véase la jurisprudencia obligatoria de la SCJN P./J. 10/2016 (10a.), Gaceta del Semanario Fudicial de la Federación, libro 34, t. I, p. 8, septiembre de 2016, registro 2012589.

35 Sobre el reconocimiento de la protección a la salud y la vida como finalidades constitucionalmente válidas que justifica interferencias en derechos fundamentales o tratos diferenciados, véanse las tesis 1a./J. 7/2019 (10a.) (Gaceta del Semanario Fudicial de la Federación, libro 63, t. I, p. 495, febrero de 2019, registro 2019381), 2a. XCII/2017 (10a.) (Gaceta del Semanario Fudicial de la Federación, libro 43, t. II, p. 1423, junio de 2017, registro 2014440), P./J. 27/2011, Semanario Fudicial de la Federación y su Gaceta, t. XXXIV, agosto de 201 1, p. 19. 
Este libro forma parte del acervo de la Biblioteca Jurídica Virtual del Instituto de Investigaciones Jurídicas de la UNAM

diabetes, etc.) que podrían resultar mucho más estigmatizantes desde un punto de vista jurídico y que, además, serían menos efectivas desde un punto de vista técnico (pues son menos adecuadas para determinar la probabilidad de éxito).

Finalmente, conviene hacer algunos apuntes sobre la forma en que convendría establecer los criterios propuestos. Ante el desequilibrio entre las necesidades clínicas y la disponibilidad efectiva de los recursos sanitarios, resulta fundamental que el modelo de triage sea actualizado en la Guía Bioética para Asignación de Recursos Limitados de Medicina Crítica en Situación de Emergencia por el Consejo de Salubridad General, toda vez que se trata de un órgano con la facultad constitucional de establecer medidas obligatorias para todas las autoridades del país y que sean necesarias para prevenir y combatir epidemias. ${ }^{36}$ Específicamente, proponemos que la discusión de los criterios se retome en la Comisión de Ética, de tal forma que sea posible emitir lineamientos vinculantes de atención médica para todas las unidades de salud del país, a fin de salvar el mayor número de vidas posible en México.

Actualizar las medidas de triage con modelos que puedan resultar más efectivos desde el Consejo de Salubridad General para la atención de la COVID-19 en México no sólo dará una mejor guía a los profesionales de salud que están haciendo frente a la pandemia, sino que establecería criterios claros y obligatorios de priorización de pacientes y de maximización de recursos disponibles para salvar el mayor número posible de vidas, en el caso específico de SARS-CoV-2.

\section{GONCLUSIONES}

El gobierno mexicano tiene la obligación de garantizar el ejercicio pleno de derechos y agotar todos los recursos disponibles para ello. El principio de universalidad que rige a todos los derechos humanos en México es una exigencia que se debe de garantizar para que, incluso en escenarios de crisis, la desigualdad y la corrupción no se impongan en el ejercicio de derechos ni en la distribución justa y equitativa de bienes y servicios públicos que, en medio de la pandemia, se hicieron aún más escasos.

Sobra decirlo, pero no estamos frente a un reto menor. Por desgracia, se han perdido muchas vidas a causa de la COVID-19. Esto es inevitable. Pero es igualmente cierto que todas las autoridades están obligadas a garantizar al máximo el derecho a la salud y a la vida de todos, sin mayor límite que las

36 En términos de la fracción XVI del artículo 73 de la Constitución. 
Este libro forma parte del acervo de la Biblioteca Jurídica Virtual del Instituto de Investigaciones Jurídicas de la UNAM

posibilidades fácticas y jurídicas. El esquema que hemos propuesto permite cumplir precisamente con ese mandato: garantizar, en la mayor medida posible, los derechos de todos.

Es posible predecir con precisión y usando herramientas disponibles en todos los hospitales en el país la mortalidad de pacientes con COVID al momento de su hospitalización. Es crucial desarrollar e implementar herramientas específicas a este contexto y basadas en evidencia científica para guiar la alocación de recursos materiales y humanos de forma eficiente y sin caer en prácticas discriminatorias.

Aprovechemos la ventana de oportunidad que dejó abierto el Comité de Ética para superar el modelo propuesto en la Guía Bioética en caso de que un modelo distinto fuese validado con información específica para pacientes COVID-19. El modelo que aquí hemos reiterado y validado ofrece una escala mucho más precisa y que brinda mayor seguridad para tomar decisiones de asignación de recursos médicos escasos. El modelo, como hemos comentado anteriormente, ya fue aceptado en el Colegio Americano de Medicina de Urgencias y por el Repositorio MedRxiv de Yale. Tal vez sea tiempo de reevaluar. Aún estamos a tiempo para salvar una enorme cantidad de vidas. 